\title{
Innovation by Evolution: Bringing New Chemistry to Life Frances Arnold ${ }^{1}$ \\ ${ }^{1}$ California Institute of Technology \\ frances@cheme.caltech.edu
}

Not satisfied with biology's vast catalyst repertoire, we create new enzyme catalysts and expand the chemistry of life. We use the most powerful biological design process, evolution, to optimize existing enzymes and invent new ones, thereby circumventing our profound ignorance of how sequence encodes function. With a little insight from chemistry, we can generate enzymes for transformations that are unknown in biology. These new-to-nature enzymes increase the scope of molecules and materials we can build using synthetic biology and move us closer to a sustainable world where human-invented chemistry will be genetically encoded. 